'Laboratorio de Oncología y Genética Molecular. Unidad de Coloproctología. Clínica Las Condes, Santiago, Chile. ${ }^{2}$ Dirección Académica. Clínica Las Condes, Santiago, Chile. ${ }^{3}$ Sub-dirección de Investigación. Latin American Collaborative Research Center, Tokyo Medical and Dental University, Santiago, Chile.

${ }^{4}$ Department of Human Pathology, Tokyo Medical and Dental University Graduate School, Tokyo, Japan. ${ }^{5}$ Servicio de Patología, Clínica Las Condes, Santiago, Chile. aDoctor en Ciencias Biológicas (PhD). bBioquímico. ‘Enfermera Universitaria, MScc Epidemiología. dEstudiante pregrado medicina. Tokyo Medical and Dental University, Tokyo, Japón.

Financiado por: Dirección Académica, Clínica Las Condes y Tokyo Medical and Dental University (TMDU).

Conflictos de intereses: Los autores declararon no tenerlos en este manuscrito.

Recibido el 20 marzo de 2014, aceptado el 9 de enero de 2015.

Correspondencia a: Claudia Hurtado R., PhD. Laboratorio de Oncología y Genética Molecular. Clínica Las Condes. Lo Fontecilla 174. (CP: 7591018)

Las Condes. Santiago, Chile. Teléfono: +56-2-22104771;

Fax: +56-2-22104776. churtado@clc.cl

\section{Análisis molecular del cáncer de colon esporádico}

\author{
CLAUDIA HURTADO ${ }^{1, \mathrm{a}}$, ANA MARÍA WIELANDT ${ }^{1, \mathrm{~b}}$, \\ ALEJANDRO J. ZÁRATE ${ }^{1}$, UDO KRONBERG ${ }^{1}$, MAGDALENA CASTRO ${ }^{2, c}$, \\ KEN YAMAGIWA ${ }^{3, \mathrm{~d}}$, TAKASHI ITO ${ }^{3}$, YOSHINOBU EISHI ${ }^{4}$, \\ LUIS CONTRERAS ${ }^{5}$, FRANCISCO LÓPEZ-KÖSTNER ${ }^{1}$
}

\section{Molecular analysis of sporadic colon cancer}

Background: In Chile, colorectal cancer (CRC) is often diagnosed in late stages. Thus, surgical treatment must be complemented with chemotherapy. KRAS mutations and microsatellite instability have been detected in these tumors. However, the response to treatment in patients without KRAS mutations varies and requires a better understanding. Aim: To determine the frequency and distribution of somatic point mutations in KRAS, BRAF and PIK3CA genes and microsatellite instability status (MSI) in patients with colon cancer (CC). Material and Methods: A prospective observational study of patients undergoing surgery for colon cancer. Tumor-derived DNA was analyzed by polymerase chain reaction (PCR) for the most frequent mutations of KRAS, BRAF and PIK3CA. PCR was also used to analyze MSI. Results: Fifty-eight patients with sporadic CC were analyzed, 16 showed KRAS mutations (G12R, G12D, G12V, G13D) and out of the 42 patients that did not show any mutation, 10 had mutations in BRAF (V600E) and PIK3CA (E542K, E545D, E545K, Q546E, H1047R). BRAF mutations alone or in combination with PIK3CA mutations were observed in $27 \%$ of high MSI tumors and in $2 \%$ of tumors without instability $(p<0.049)$. A higher percentage of high MSI tumors were located in the right colon $(p<0.001)$, and showed BRAF mutation $(p<0.020)$. Conclusions: The highest percentage of high MSI and BRAF mutations was observed in the right colon. Therefore, this study suggests the presence of different molecular features between right and left colon tumors that should be considered when defining the therapeutic management.

(Rev Med Chile 2015; 143: 310-319)

Key words: BRAF protein, human; Colonic neoplasms; KRAS protein, human; PIK3CA protein, human.
1 1 cáncer colorrectal (CCR) es una de las causas de mortalidad por cáncer más frecuentes en los países desarrollados ${ }^{1}$. En Chile, el CCR ha presentado un aumento significativo en los últimos años, tanto en hombres como en mujeres ${ }^{2,3}$.

La mayoría de los pacientes con cáncer de colon (CC) avanzado son tratados con cirugía resectiva, seguida de esquemas de quimioterapia según corresponda. Las quimioterapias más usa- das están basadas en fluorouracilo, leucovorina y oxaliplatino (FOLFOX) o irinotecan (FOLFIRI). Los pacientes con enfermedad metastásica también pueden ser tratados con terapias basadas en anticuerpos monoclonales, como panitumumab o cetuximab, que han demostrado mejorar la supervivencia libre de progresión y la supervivencia global, mediante el bloqueo de la señalización del receptor del factor de crecimiento epidermal (EGFR). Esta vía tiene un rol importante en la 
patogénesis del $\mathrm{CCR}^{4,5}$, en la cual participan las vías de las MAPK (proteína quinasa activada por mitógeno) y PI3K (proteína fosfoinositol 3-quinasa)/Akt. Esta activación promueve procesos claves como la transcripción, migración, angiogénesis, crecimiento celular y apoptosis ${ }^{6}$.

En cuanto a la eficacia de los anticuerpos, ésta es variable, observándose que algunos pacientes no presentan una respuesta favorable al administrar tratamientos anti-EGFR. Esto se explica en gran medida por la presencia de mutaciones activantes en ciertos genes pertenecientes a esta vía, que confieren resistencia a la terapia. Entre estos, han sido blancos de estudio los genes KRAS, BRAF y PIK3CA. Se ha reportado que en pacientes sin mutación del gen KRAS, la tasa de respuesta a la terapia monoclonal puede ser de 9 a $28 \%{ }^{7}$; lo cual se explica por la presencia de mutaciones activantes en otros genes como BRAF (codón 600) o PIK3CA (subunidad $\mathrm{p} 110 \alpha)^{8-10}$.

La frecuencia de mutaciones en KRAS, BRAF y PIK3CA ha sido reportada en otras poblaciones con CCR (Bélgica, Alemania, Corea, Noruega, España, Italia, entre otros $)^{11-17}$. No obstante, hay escasos reportes de la frecuencia de estas mutaciones en Latinoamérica.

Ciardello et $\mathrm{ll}^{18}$ reportan que la frecuencia de mutaciones de KRAS en América Latina es de $36 \%$, lo cual es similar a Europa (40\%) y mayor que en Asia (22\%). Otro estudio realizado en población peruana determinó que la frecuencia de mutaciones de KRAS y BRAF es de $16,7 \%$ y $9,9 \%$ respectivamente ${ }^{19}$.

Otra característica importante es la inestabilidad de microsatélites (MSI), que constituye otro factor pronóstico y predictivo en los pacientes con $\mathrm{CCR}^{20}$. Se ha observado que pacientes con CCR en estadío II y MSI alta, el uso de 5-fluorouracilo no beneficiaría al paciente, sugiriendo al estado de MSI como un valor predictivo de respuesta a terapia; por otra parte, se le ha otorgado un valor de mal pronóstico a la ausencia de MSI junto a la presencia de mutaciones en BRAF en pacientes con $\mathrm{CCR}^{21-24}$. En Chile no hay estudios que analicen en conjunto los genes KRAS, BRAF y PIK3CA y la MSI en pacientes con CC. Lo anterior tiene relevancia al momento de conocer las opciones para determinadas terapias en estos pacientes. Dado que las variables moleculares más frecuentemente utilizadas son la determinación del estado mutacional de KRAS y la MSI, el objetivo del trabajo es analizar la frecuencia y distribución de mutaciones somáticas puntuales en los genes KRAS, BRAF y PIK3CA y el estado de la MSI en pacientes con CC esporádico.

\section{Materiales y Métodos}

\section{Diseño del estudio}

Estudio observacional prospectivo realizado durante 2 años, en el cual se reclutaron pacientes mayores de 18 años con diagnóstico de adenocarcinoma de colon. Se excluyeron los pacientes con cáncer de recto y con historia familiar de CC. Cada paciente firmó un consentimiento informado que fue previamente aprobado por el Comité de Bioética de Clínica Las Condes. Los datos clínicos, demográficos e histopatológico de todos los pacientes fueron incluidos en una base de datos.

\section{Extracción de ADN tumoral}

Las secciones teñidas con hematoxilina-eosina de las muestras fijadas en formalina e incluídas en parafina (FFPE) fueron revisadas por el médico patólogo institucional quien seleccionó áreas con al menos $80 \%$ de células tumorales. Luego, se sacó un trozo del área seleccionada y se le realizó una nueva inclusión. De él se hicieron cortes seriados de 8 micrones de espesor no teñidos y se extrajo el ADN usando el kit QIAGEN QIAmp DNA FFPE Tissue ${ }^{\circledR}$ de acuerdo a las instrucciones del fabricante. La integridad del ADN se analizó mediante la amplificación por PCR multiplex de 2 amplicones de GAPDH, 1 amplicón de $\beta$-globina y 1 amplicón de $\beta$-actina ${ }^{25}$. Se utilizaron sólo las muestras que amplificaban a lo menos 300 pb. Para determinar el estado de MSI de los pacientes, el ADN control se extrajo a partir de muestras de sangre del paciente, utilizando el método descrito por Lahiri y Nurnberger ${ }^{26}$.

\section{Análisis de mutaciones somáticas en KRAS, BRAF $y$ PIK3CA}

El ADN del tumor se analizó mediante reacción en cadena de la polimerasa (PCR) para las mutaciones más frecuentes de cada gen. Los partidores fueron diseñados para analizar los codones 12 y 13 del exón 2 de KRAS, el codón 600 de BRAF, y los exones 9 y 20 de PIK3CA. Para evitar la amplificación del pseudogen de PIK3CA, se diseñó un partidor específico para el 
exón 9. El listado de partidores y condiciones de las reacciones de PCR se detallan en la Tabla 1. La amplificación de los exones se realizó utilizando la enzima Platinum ${ }^{\circledR}$ TAQ DNA polymerase (Life Technologies) y un programa de 35 ciclos, utilizando una temperatura de alineamiento de $55^{\circ} \mathrm{C}$ para los genes KRAS y PIK3CA y de $58^{\circ} \mathrm{C}$ para el gen BRAF en un termociclador Applied Biosystems 2720 (Life Technologies). Los productos de PCR se visualizaron en geles de agarosa al $2 \%$, teñidos con bromuro de etidio. Los genes KRAS y PIK3CA se analizaron por SSCP (Single Strand Conformomer Polymorphisms) y por secuenciación dirigida mediante el secuenciador automático ABI PRISM 3100. Se confirmaron todas las mutaciones encontradas secuenciando ambas hebras del $\mathrm{ADN}$. El gen BRAF se estudió directamente por secuenciación.

\section{Análisis del estado de MSI}

Se determinó mediante la amplificación por PCR de los cinco marcadores microsatelitales recomendados por el National Cancer Institute (NCI) de los Estados Unidos de Norteamérica para el CCR: los mononucléotidos Bat-25, Bat-26 y los dinucleótidos D2S123, D3S1029, D5S346 ${ }^{27,28}$. Cada marcador microsatelital fue amplificado a partir de ADN tumoral y ADN control utilizando partidores marcados con fluoróforos según lo descrito por Raptis y cols, $2007^{29}$. La reacción de PCR fue realizada con 40 ciclos a $55^{\circ} \mathrm{C}$ en un volumen final de $25 \mu \mathrm{l}$. Los microsatélites amplificados fueron separados por electroforesis capilar mediante el secuenciador ABI 310. Los resultados de la separación en el secuenciador se obtuvieron mediante el software Gene Mapper 4.0 de Applied Biosystems. Los tumores con 2 o más microsatélites inestables $(>30 \%)$ y/o alteración en el patrón de migración son considerados como MSI-alta, tumores con sólo un microsatélite inestable son considerados como MSI-baja y los tumores sin microsatélites inestables se consideran como estables (MSS) ${ }^{21}$. Clínicamente se ha establecido que la MSI-baja se comporta como MSS, por lo que en este trabajo se considerarán como un solo $\operatorname{grupo}^{29,30}$.

\section{Análisis estadístico}

Se obtuvieron las variables demográficas y las características histopatológicas del tumor como el tipo histológico, la diferenciación del tumor y estadio de acuerdo a la clasificación de 2011 de la American Joint Cancer Committee/Union Internationale Contre le Cancer (AJCC/UICC). Se analizaron las mutaciones en KRAS, BRAF y PIK3CA y el estado de MSI. Los datos categóricos se resumieron en frecuencias y porcentajes. Las asociaciones entre los datos categóricos se analizaron mediante pruebas de chi-cuadrado. Valores de $\mathrm{p}$ inferiores a 0,05 fueron considerados estadísticamente significativos.

\section{Resultados}

Se enroló un total de 58 pacientes ( 28 hombres y 30 mujeres) con CC esporádico. De estos, 30 (52\%) mujeres con una edad promedio de diag-

Tabla 1. Partidores y condiciones utilizados para la amplificación de los genes KRAS, BRAF y PIK3CA

\begin{tabular}{|c|c|c|c|c|}
\hline Nombre partidor & Secuencia & Referencia & $\begin{array}{c}\text { Tamaño } \\
\text { esperado } \\
\text { (pb) }\end{array}$ & $\begin{array}{c}\text { Temperatura de } \\
\text { alineamiento } \\
\left({ }^{\circ} \mathrm{C}\right)\end{array}$ \\
\hline KRAS e1F & 5' AAGGTACTGGTGGAGTATTTG '3 & propio & \multirow{2}{*}{253} & \multirow{2}{*}{58} \\
\hline KRAS e1R & 5' GAATGGTCCTGCACCAGTAAT '3 & propio & & \\
\hline BRAF e15F & 5' CCTAAACTCTTCATAATGCTTGCTC '3 & Propio & \multirow{2}{*}{189} & \multirow{2}{*}{55} \\
\hline BRAF e15R & 5' CCACAAAATGGATCCAGACA '3 & propio & & \\
\hline PIK3CA e9F & 5' TGCTTTTTCTGTAAATCATCTGT '3 & propio & \multirow{2}{*}{289} & \multirow{2}{*}{55} \\
\hline PIK3CA e9R específico & 5' CATTTTAGCACTTACCTGTGA '3 & propio & & \\
\hline PIK3CA e20F & 5' TATTCGACAGCATGCCAATCT '3 & propio & \multirow{2}{*}{348} & \multirow{2}{*}{55} \\
\hline PIK3CA e20R & 5' ATGCTGTTCATGGATTGTGCA '3 & propio & & \\
\hline
\end{tabular}


Tabla 2. Características clínico-patológicas y el estado mutacional de los pacientes

\begin{tabular}{|c|c|c|c|c|}
\hline Características clínico-patológicas & $\begin{array}{c}\text { Total } \\
\text { n (\%) } \\
58(100 \%)\end{array}$ & $\begin{array}{c}\text { Hombres } \\
\text { n (\%) } \\
28(48 \%)\end{array}$ & $\begin{array}{l}\text { Mujeres } \\
\text { n (\%) } \\
30(52 \%)\end{array}$ & $\mathbf{p}$ \\
\hline $\begin{array}{l}\text { Edad (años) } \\
>50 \\
\leq 50\end{array}$ & $\begin{array}{l}46(79 \%) \\
12(21 \%)\end{array}$ & $\begin{array}{r}25(54 \%) \\
3(25 \%)\end{array}$ & $\begin{array}{r}21(46 \%) \\
9(75 \%)\end{array}$ & 0,07 \\
\hline $\begin{array}{l}\text { Localización } \\
\text { Derecho } \\
\text { Izquierdo }\end{array}$ & $\begin{array}{l}19(33 \%) \\
39(67 \%)\end{array}$ & $\begin{array}{r}7(37 \%) \\
21(54 \%)\end{array}$ & $\begin{array}{l}12 \text { (63\%) } \\
18(46 \%)\end{array}$ & 0,515 \\
\hline $\begin{array}{l}\text { Profundidad de la invasión } \\
\text { pT1 } \\
\text { pT2 } \\
\text { pT3 } \\
\text { pT4 }\end{array}$ & $\begin{array}{r}5(9 \%) \\
14(24 \%) \\
36(62 \%) \\
3 \quad(5 \%)\end{array}$ & $\begin{array}{r}4(80 \%) \\
5(36 \%) \\
17(47 \%) \\
2(67 \%)\end{array}$ & $\begin{array}{r}1(20 \%) \\
9(64 \%) \\
19(53 \%) \\
1(33 \%)\end{array}$ & 0,404 \\
\hline $\begin{array}{l}\text { Estado linfonodal } \\
+ \\
-\end{array}$ & $\begin{array}{l}24(41 \%) \\
34(59 \%)\end{array}$ & $\begin{array}{l}12(50 \%) \\
16(47 \%)\end{array}$ & $\begin{array}{l}12(50 \%) \\
18(53 \%)\end{array}$ & 0,825 \\
\hline $\begin{array}{l}\text { Grado de diferenciación } \\
\text { Bien } \\
\text { Moderado } \\
\text { Pobre }\end{array}$ & $\begin{array}{rr}1 & (2 \%) \\
52 & (90 \%) \\
5 & (8 \%)\end{array}$ & $\begin{array}{r}0(0 \%) \\
25(48 \%) \\
3(60 \%)\end{array}$ & $\begin{array}{r}1(100 \%) \\
27(52 \%) \\
2(40 \%)\end{array}$ & 0,829 \\
\hline $\begin{array}{l}\text { Etapa } \\
\text { I } \\
\text { II } \\
\text { III } \\
\text { IV }\end{array}$ & $\begin{array}{l}12(21 \%) \\
22(38 \%) \\
21(36 \%) \\
3 \quad(5 \%)\end{array}$ & $\begin{array}{r}7(58 \%) \\
9(41 \%) \\
10(48 \%) \\
2(67 \%)\end{array}$ & $\begin{array}{r}5(42 \%) \\
13(59 \%) \\
11(52 \%) \\
1(33 \%)\end{array}$ & 0,718 \\
\hline $\begin{array}{l}\text { Estado de MSI } \\
\text { MSI-baja/MSS } \\
\text { MSI-alta }\end{array}$ & $\begin{array}{l}43(74 \%) \\
15(26 \%)\end{array}$ & $\begin{array}{r}21(49 \%) \\
7(47 \%)\end{array}$ & $\begin{array}{r}22(51 \%) \\
8(53 \%)\end{array}$ & 0,885 \\
\hline $\begin{array}{l}\text { Estado de mutación } \\
\text { Mutaciones KRAS } \\
\text { Mutaciones KRAS/PIK3CA } \\
\text { Mutaciones BRAF } \\
\text { Mutaciones BRAF/PIK3CA } \\
\text { Mutaciones PIK3CA }\end{array}$ & $\begin{array}{rr}26 & (45 \%) \\
13 & (22 \%) \\
3 & (5 \%) \\
4 & (7 \%) \\
1 & (2 \%) \\
5 & (9 \%)\end{array}$ & $\begin{array}{r}12(46 \%) \\
6(46 \%) \\
1(33 \%) \\
2(50 \%) \\
1(100 \%) \\
2(40 \%)\end{array}$ & $\begin{array}{r}14(54 \%) \\
7(54 \%) \\
2(67 \%) \\
2(50 \%) \\
0(0 \%) \\
3(60 \%)\end{array}$ & 0,771 \\
\hline
\end{tabular}

nóstico de 62 años (rango: 36-90). Los hombres presentaron una edad de diagnóstico promedio de 65 años (rango: 35-87). El tumor se localizó en el colon derecho en $33 \%$ de los pacientes y $41 \%$ presentaba metástasis ganglionares al momento de la cirugía. La mayoría de los tumores fueron clasificados como moderadamente diferenciados (Tabla 2).

Se identificaron 30 mutaciones en 26 pacientes (45\%) siendo la más frecuente la del gen KRAS, que se observó en 16 pacientes (27\%). Las mutaciones encontradas en el gen KRAS fueron:
G12R, G12D, G12V y G13D. En la Figura 1 se muestran algunos ejemplos de las mutaciones identificadas. En los pacientes sin mutación de KRAS (wild-type), 10 de ellos tenían mutaciones en otros genes ( 4 en BRAF (V600E), 5 en PIK3CA (E542K, E545D, E545K, Q546E y H1047R) y 1 presentaba una doble mutación en BRAF (V600E) y PIK3CA (E545D).

Hubo 4 pacientes con mutaciones simultáneas, es decir con dos genes mutados, 3 presentaban simultáneamente mutaciones en KRAS y PIK3CA (2 pacientes con G12D y E542K y 1 paciente con 


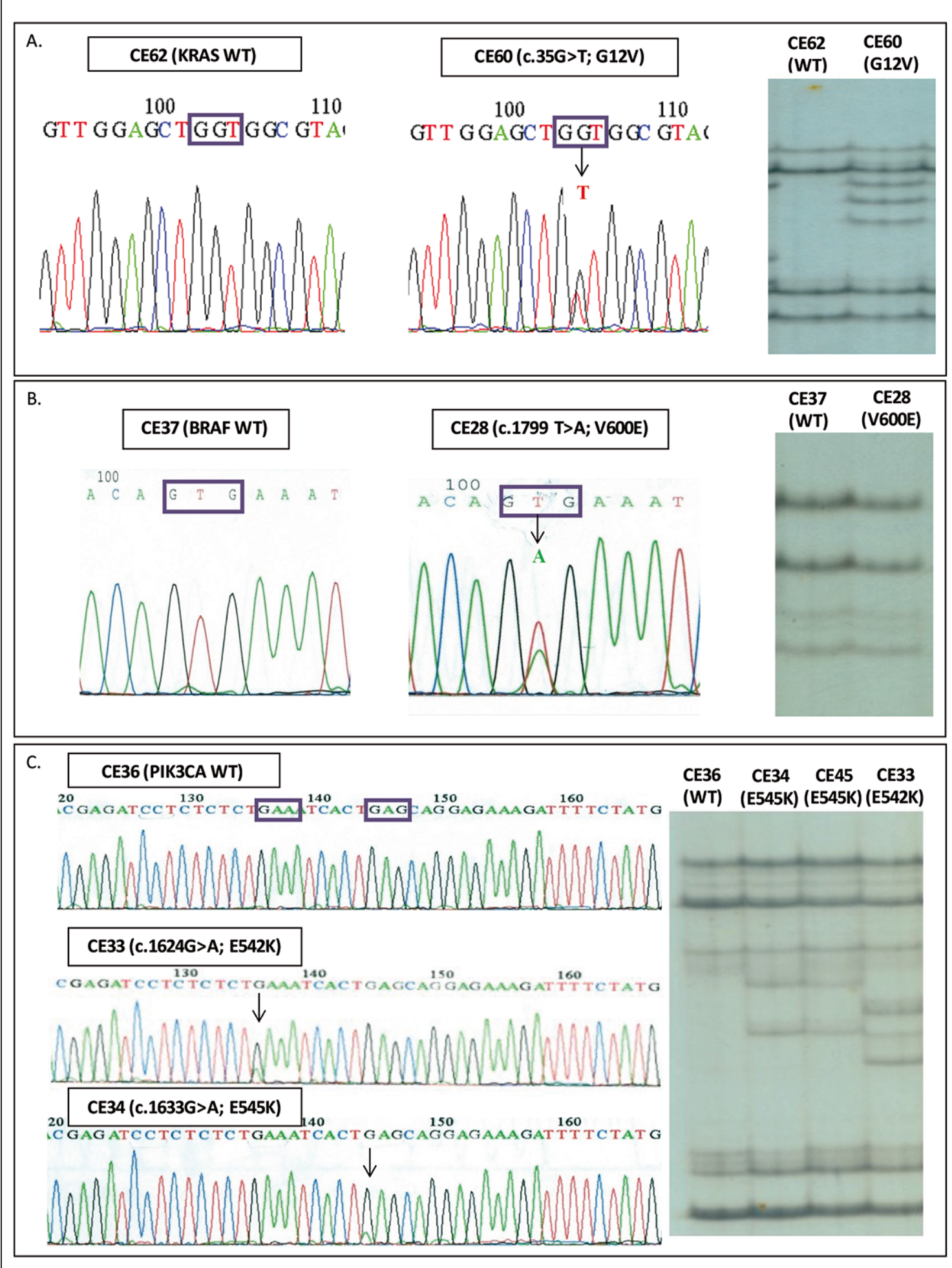

Figura 1. Electroferogramas y patrón de migración de geles de SSCP de algunas de las mutaciones identificadas. 
Tabla 3. Frecuencia de mutaciones identificadas

\begin{tabular}{|lcrrrrr|}
\hline & \multicolumn{2}{c}{ Total } & \multicolumn{2}{c|}{ Hombres } & \multicolumn{2}{c|}{ Mujeres } \\
& n (\%) & n & $(\mathbf{( \% )}$ & n & $(\mathbf{( \% )}$ & p \\
KRAS & $16(28 \%)$ & 7 & $(44 \%)$ & 9 & $(56 \%)$ & 0,536 \\
G12R & 1 & $1(100 \%)$ & 0 & $(0 \%)$ & \\
G12D & 5 & 1 & $(20 \%)$ & 4 & $(80 \%)$ & \\
G12V & 5 & 2 & $(40 \%)$ & 3 & $(60 \%)$ & \\
G13D & 5 & 3 & $(60 \%)$ & 2 & $(40 \%)$ & \\
BRAF & & & & & & 0,583 \\
V600E & $5(9 \%)$ & 3 & $(60 \%)$ & 2 & $(40 \%)$ & \\
PIK3CA & $9(16 \%)$ & 4 & $(44 \%)$ & 5 & $(56 \%)$ & 0,508 \\
E542K & 2 & 0 & $(0 \%)$ & $2(100 \%)$ & \\
E545D & 1 & $1(100 \%)$ & 0 & $(0 \%)$ & \\
E545K & 2 & 1 & $(50 \%)$ & 1 & $(50 \%)$ & \\
Q546E & 1 & 0 & $(0 \%)$ & $1(100 \%)$ & \\
H1047R & 3 & 2 & $(67 \%)$ & 1 & $(33 \%)$ & \\
\hline
\end{tabular}

G12V y E545K), sin MSI, estadio II. El cuarto paciente presentaba simultáneamente mutaciones en BRAF (V600E) y PIK3CA (E545D) con MSI alta y estadio III. Todos ellos tuvieron tumores ubicados en el colon izquierdo.

No hubo pacientes con mutaciones simultáneas en KRAS y BRAF. La frecuencia específica de mutaciones encontradas se muestra en la Tabla 3.

Respecto de la MSI, 26\% (15/58) de los tumores presentaba MSI alta y el porcentaje de pacientes con MSI alta no varió según género (Tabla 2). Hubo 30\% de MSI alta en mayores de 50 años, en comparación a $8 \%$ en menores de 50 años $(\mathrm{p}=0,119)$. En cuanto a la localización de los tumores, $58 \%$ de los tumores de colon derecho demostró MSI alta (11/19), comparado con $10 \%$ de los tumores del lado izquierdo (4/39;

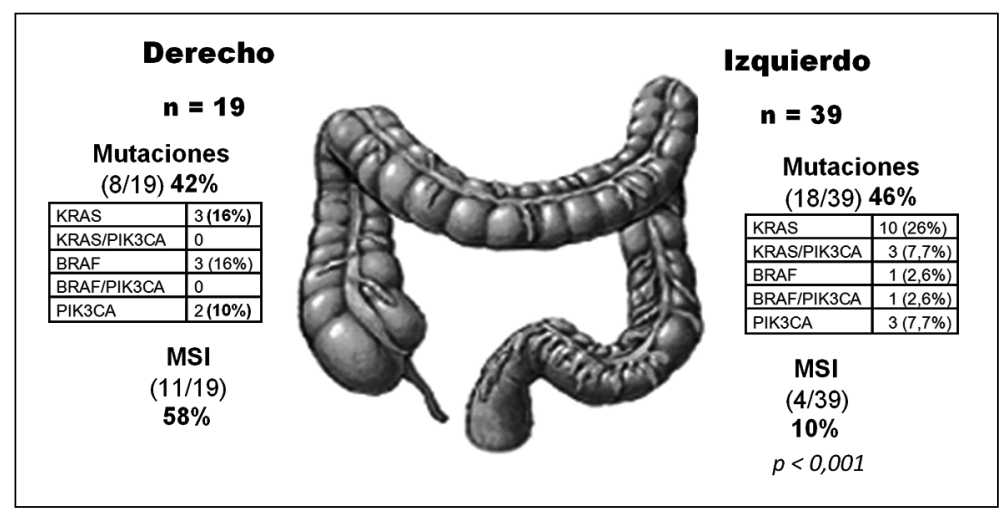

Figura 2. Distribución de mutaciones y MSI según la localización del tumor.

Tabla 4. Distribución de mutaciones en pacientes según la etapa y estado de inestabilidad microsatelital (MSI)

\begin{tabular}{|c|c|c|c|c|c|c|c|}
\hline \multirow[b]{2}{*}{ Mutaciones } & \multirow[b]{2}{*}{$\begin{array}{c}\text { n de pacientes } \\
\qquad \begin{array}{c}\mathbf{n}=\mathbf{5 8} \\
\mathbf{n} \quad(\%)\end{array}\end{array}$} & \multicolumn{2}{|c|}{ Etapa } & \multicolumn{4}{|c|}{ Estado MSI } \\
\hline & & $\begin{array}{c}\text { I-II } \\
n=34\end{array}$ & $\begin{array}{l}\text { III-IV } \\
n=24\end{array}$ & $\mathbf{p}$ & $\begin{array}{l}\text { MSI-alta } \\
n=15\end{array}$ & $\begin{array}{c}\text { MSI-baja/ } \\
\text { MSS } \\
n=43\end{array}$ & $\mathbf{p}$ \\
\hline KRAS & 13 & $8(23 \%)$ & $5(21 \%)$ & 0,808 & $3(20 \%)$ & $10(23 \%)$ & 0,795 \\
\hline KRAS/PIK3CA & 3 & $3(9 \%)$ & $0 \quad(0 \%)$ & 0,135 & $0 \quad(0 \%)$ & $3(7 \%)$ & 0,293 \\
\hline BRAF & 4 & $2(6 \%)$ & $2(8 \%)$ & 0,717 & $3(20 \%)$ & $1 \quad(2 \%)$ & $0,020^{*}$ \\
\hline BRAF/PIK3CA & 1 & $0 \quad(0 \%)$ & 1 (4\%) & 0,230 & $1 \quad(7 \%)$ & $0 \quad(0 \%)$ & 0,088 \\
\hline PIK3CA & 5 & $4(12 \%)$ & $1 \quad(4 \%)$ & 0,310 & $3(20 \%)$ & $2(5 \%)$ & 0,068 \\
\hline Total & $26(45 \%)$ & $17(50 \%)$ & $9(37 \%)$ & 0,340 & $10(67 \%)$ & $16(37 \%)$ & 0,885 \\
\hline Wild-type & 32 & 17 (50\%) & $15(63 \%)$ & --- & $5(33 \%)$ & $27(63 \%)$ & --- \\
\hline MSI-alta & 15 & $8(24 \%)$ & $7(29 \%)$ & 0,629 & --- & --- & --- \\
\hline
\end{tabular}

Abreviaturas: MSI-baja/MSS, inestabilidad baja/estable; MSI-alta, inestabilidad-alta. Nota: *significancia estadística. 
$\mathrm{p}<0,001$ ) (Figura 2). Además, en la Figura 2 se observa un porcentaje de mutaciones diferentes según su localización entre los genes KRAS, BRAF y PIK3CA, con una tendencia de BRAF asociado a colon derecho y KRAS a colon izquierdo que es independiente del estado linfonodal.

La mutación de KRAS sola o en combinación con PIK3CA estuvo presente en $20 \%$ de las muestras de MSI alta y $30 \%$ de las MSI baja/MSS. La mutación de BRAF sola o en combinación con PIK3CA en tumores MSI alta fue 27\% y $2 \%$ de los MSI baja/MSS ( $<$ 0,049) (Tabla 4).

La distribución de mutaciones según la etapa tumoral (I-II versus III-IV) mostró una mayor tasa en etapas tempranas (I-II), comparadas con la etapa tardía, sin alcanzar significación estadística $(50 \%$ versus $37 \%$; $\mathrm{p}=0,34)($ Tabla 4$)$.

\section{Discusión}

En este estudio, 45\% (26/58) de los pacientes con CC presentó al menos una mutación de los genes KRAS, BRAF o PIK3CA. Esto evidencia que la frecuencia de mutaciones de los genes mencionados se enmarca dentro de los rangos descritos en América del Norte y Europa ${ }^{8,11-17}$, a diferencia de estudios como el realizado en Perú ${ }^{19}$, que da cuenta de una menor frecuencia de mutaciones en el gen KRAS, que según los autores es atribuido a una multietnicidad de su población.

En Chile, Roa et al., recientemente han descrito sobre $40 \%$ de mutaciones en KRAS y $15 \%$ de mutaciones en $\mathrm{BRAF}^{31,32}$, diferente a lo observado en este estudio ( $27 \%$ y $9 \%$, respectivamente). Estas diferencias pueden deberse a nuestro limitado tamaño muestral, sin embargo, coincidimos al observar la misma frecuencia de distribución del tipo de mutación identificada.

En el presente trabajo, los tumores con mutaciones en BRAF se asociaron significativamente a MSI alta y localización en el colon derecho, lo cual es consistente con reportes previos ${ }^{33,34}$. Aun más, ha sido reportado que la mutación activante en BRAF fue encontrada entre $10-18 \%$ de pacientes con CC y que este porcentaje se incrementa a 27$45 \%$ en quienes presentan tumores MSI alta ${ }^{35,36}$, en concordancia nosotros observamos $9 \%$ de mutación en BRAF en nuestra población de estudio y que alcanza a $27 \%$ en aquellos con MSI alta. Respecto de las mutaciones, la mutación en BRAF está asociada a carcinomas avanzados y raramente se presenta en tumores MSS ${ }^{37}$.

Se ha propuesto que la existencia de tumores con BRAF mutado y MSI alta se asociaría a una vía carcinogénica diferente de la clásica adenoma a carcinoma ${ }^{38}$. Si bien las mutaciones en BRAF han sido reportadas como indicadores de mal pronóstico en pacientes con tumores de colon derecho y/o MSS $^{32,9}$, la presencia de mutación en BRAF podría tener un valor predictivo ya que terapias específicas como vemurafenib (PLX 4032) y GSK 2118436, usados actualmente para pacientes con melanoma que tienen mutación en el gen BRAF, podrían ser usados también en pacientes con $\mathrm{CC}^{39}$.

En este estudio se observó que las mutaciones en BRAF fueron excluyentes con las de KRAS, lo cual ya está establecido en la literatura ${ }^{33}$, por esta razón para una mejor caracterización molecular ambos marcadores deberían ser considerados en los análisis.

La mutación activante de KRAS fue encontrada en proporciones similares a las descritas por Oliveira y cols. ${ }^{40}$ en pacientes con tumores MSI alta y MSI baja-MSS, quienes demostraron que éstas estaban presente en $22 \%$ de las MSI alta y $34 \%$ de las MSS $^{37,40}$. En el caso particular de la mutación activante G13D, esta se observó en 5 de los $58(8,6 \%)$ pacientes estudiados. En los últimos años ha existido controversia con respecto a que la mutación G13D sería de mejor pronóstico y con mejor respuesta a terapia que mutaciones en el codón 12, sin embargo, estudios recientes han demostrado lo contrario ${ }^{33,41-44}$.

Con respecto a las alteraciones funcionales que provocarían las mutaciones descritas en este trabajo, estas serían de carácter oncogénicas, ya que por ejemplo las mutaciones en los codones 12 y 13 del gen KRAS provocan cambios en el loop-P GTP (guanosina trifosfato) previniendo la hidrólisis de GTP y, por ende, activando así permanentemente moléculas de $\mathrm{RAS}^{45}$. Por su parte, la mutación en el codón 600 del gen BRAF resulta en la activación constitutiva de la proteína debida a que el cambio de valina $(\mathrm{V})$ por glutámico (E) imita la fosforilación de otros aminoácidos como T599 o S602 en el segmento de activación. Este cambio provoca que la proteína BRAF se mantenga constitutivamente activa de manera independiente de $\mathrm{KRAS}^{46}$. En cuanto a las mutaciones de PIK3CA, estas han sido identificadas en diferentes tipos de tumores ${ }^{45}$. Las mutaciones en este gen llevan a una activación 
constitutiva de la actividad enzimática de $\mathrm{p} 110 \alpha$, lo que estimula la vía de señalización de AKT y permite crecimiento de manera independiente de factores de crecimiento ${ }^{47}$.

En este estudio se identificaron 4 tipos de mutaciones en el exón 9 y una en el exón 20, dando cuenta de $16 \%$ de todas las mutaciones. En pacientes con CC, las mutaciones de PIK3CA, solas o en combinación con mutaciones de KRAS o BRAF, están presentes en 14 a $25 \%$ de los $\operatorname{casos}^{48,49}$, independiente del estado de la MSI ${ }^{49}$. Además, Velho et al. ${ }^{49,50}$ reportaron que en CC, las mutaciones de PIK3CA pueden ocurrir concomitantemente con mutaciones de KRAS y BRAF en pacientes con MSI alta y MSS, similar a lo descrito en este estudio.

Por otro lado, en un reciente estudio de Liao y cols., observaron que los pacientes que tenían tumores con mutación en PIK3CA y que tomaban aspirina presentaron una mayor sobrevida que aquellos sin mutación, pero no así los pacientes con tumores que tienen mutación en BRAF. Aunque estos datos son muy interesantes deben considerarse como preliminares y requieren validación a futuro, dado el pequeño número de pacientes incluidos en ese estudio ${ }^{51-53}$.

Con respecto al tratamiento de pacientes con CC y el estado de estabilidad/inestabilidad microsatelital, los pacientes con tumores MSI alta han mostrado una sensibilidad diferente a los agentes quimioterapéuticos, apareciendo como más resistentes al cisplatino, carboplatino y el 5 fluorouracilo $(5-\mathrm{FU})^{54,55}$, pero sensibles al irinotecán ${ }^{56}$. Por tanto, se ha propuesto considerar el estatus de inestabilidad microsatelital para definir la terapia en estos pacientes.

Actualmente la mayoría de los centros realizan solamente el análisis de KRAS para determinar qué pacientes serán susceptibles para responder a la terapia anti-EGFR. Sin embargo, alrededor de 9 a $28 \%$ de los pacientes sin mutación en el gen KRAS fallan en la respuesta a la terapia mencionada ${ }^{7}$, posiblemente debido a la heterogeneidad de las células tumorales del CC, dada por la presencia de mutaciones en otros genes de la vía.

En conclusión, en este estudio 24\% (10/42) de los pacientes sin mutación en el gen KRAS presenta mutaciones en BRAF o PIK3CA, por lo que incluir el análisis de esos genes permitiría una mejor selección de terapias más específicas. La presencia de mutaciones de KRAS, BRAF y PIK3CA es similar a lo observado en otros estudios incluso de diferentes zonas geográficas. Asimismo, BRAF y KRAS son mutuamente excluyentes y hay mayor porcentaje de MSI y mutación en BRAF en el colon derecho. Por todo ello, este estudio sugiere que existen características moleculares diferentes entre los tumores de colon derecho e izquierdo que deben considerarse al definir la conducta terapéutica.

\section{Referencias}

1. Siegel R, Naishadham D, Jemal A. Cancer statistics, 2012. CA Cancer J Clin 2012; 62: 10-29.

2. Información disponible en: www.deis.cl. Último acceso 2014.

3. Zárate AJ, Alonso FT, Garmendia ML, López-Köstner F. Increasing crude and adjusted mortality rates for colorectal cancer in a developing South American country. Colorectal Dis 2013; 15: 47-51.

4. Heinemann V, Stintzing S, Kirchner T, Boeck S, Jung A. Clinical relevance of EGFR- and KRAS-status in colorectal cancer patients treated with monoclonal antibodies directed against the EGFR. Cancer Treat Rev 2009; 35: 262-71.

5. Petrelli F, Borgonovo K, Cabiddu M, Ghilardi M, Barni S. Cetuximab and panitumumab in KRAS wild-type colorectal cancer: a meta-analysis. Int J Colorectal Dis 2011; 26: 823-33.

6. Di Fiore F, Sesboüé R, Michel P, Sabourin JC, Frebourg T. Molecular determinants of anti-EGFR sensitivity and resistance in metastatic colorectal cancer. $\mathrm{Br} \mathrm{J}$ Cancer 2010; 103: 1765-72.

7. Peeters M, Price T, Van Laethem JL. Anti-epidermal growth factor receptor monotherapy in the treatment of metastatic colorectal cancer: where are we today? Oncologist 2009; 14: 29-39.

8. Baldus SE, Schaefer KL, Engers R, Hartleb D, Stoecklein NH, Gabbert HE. Prevalence and heterogeneity of KRAS, BRAF, and PIK3CA mutations in primary colorectal adenocarcinomas and their corresponding metastases. Clin Cancer Res 2010; 16: 790-9.

9. Samowitz WS, Sweeney C, Herrick J, Albertsen H, Levin TR, Murtaugh MA, et al. Poor survival associated with the BRAF V600E mutation in microsatellite-stable colon cancers. Cancer Res 2005; 65: 6063-9.

10. Ogino S, Nosho K, Kirkner GJ, Shima K, Irahara N, Kure $\mathrm{S}$. PIK3CA mutation is associated with poor prognosis among patients with curatively resected colon cancer. J Clin Oncol 2009; 27: 1477-84.

11. De Roock W, Claes B, Bernasconi D, De Schutter J, 
Biesmans B, Fountzilas G, et al. Effects of KRAS, BRAF, NRAS, and PIK3CA mutations on the efficacy of cetuximab plus chemotherapy in chemotherapy-refractory metastatic colorectal cancer: a retrospective consortium analysis. Lancet Oncol 2010; 11: 753-62.

12. Balschun K, Haag J, Wenke AK, von Schönfels W, Schwarz NT, Röcken C. KRAS, NRAS, PIK3CA exon 20, and BRAF genotypes in synchronous and metachronous primary colorectal cancers diagnostic and therapeutic implications. J Mol Diagn 2011; 13: 436-45.

13. Kwon MJ, Lee SE, Kang SY, Choi YL. Frequency of KRAS, BRAF, and PIK3CA mutations in advanced colorectal cancers: Comparison of peptide nucleic acid-mediated PCR clamping and direct sequencing in formalin-fixed, paraffin-embedded tissue. Pathol Res Pract 2011; 207: 762-8.

14. László L. Predictive and prognostic factors in the complex treatment of patients with colorectal cancer. Magy Onkol 2010; 54: 383-94.

15. Berg M, Danielsen SA, Ahlquist T, Merok MA, Ågesen $\mathrm{TH}$, Vatn MH. DNA sequence profiles of the colorectal cancer critical gene set KRAS-BRAF-PIK3CA-PTENTP53 related to age at disease onset. PLoS One 2010; 5: e13978.

16. Herreros-Villanueva M, Gómez-Manero N, Muñiz P, García-Girón C, Coma del Corral MJ. PIK3CA mutations in KRAS and BRAF wild type colorectal cancer patients. A study of Spanish population. Mol Biol Rep 2011; 38: 1347-51.

17. Simi L, Pratesi N, Vignoli M, Sestini R, Cianchi F, Valanzano R. High-resolution melting analysis for rapid detection of KRAS, BRAF, and PIK3CA gene mutations in colorectal cancer. Am J Clin Pathol 2008; 130: 24753.

18. Ciardiello F, Tejpar S, Normanno N, Mercadante D, Teague $\mathrm{T}$, Wohlschlegel $\mathrm{B}$, et al. Uptake of KRAS mutation testing in patients with metastatic colorectal cancer in Europe, Latin America and Asia. Target Oncol 2011; 6: 133-45.

19. Egoavil CM, Montenegro P, Soto JL, Casanova L, Sánchez-Lihon J, Castillejo MI, et al. Clinically important molecular features of Peruvian colorectal tumours: high prevalence of DNA mismatch repair deficiency and low incidence of KRAS mutations. Pathology 2011; 43: 22833.

20. Vilar E, Gruber SB. Microsatellite instability in colorectal cancer-the stable evidence. Nat Rev Clin Oncol 2010; (3): 153-62. Review.

21. Di Nicolantonio F, Martini M, Molinari F, SartoreBianchi A, Arena S, Saletti P, et al. Wild-type BRAF is required for response to panitumumab or cetuximab in metastatic colorectal cancer. J Clin Oncol 2002; 6 (35): 5705-12.

22. Roth AD, Tejpar S, Delorenzi M, Yan P, Fiocca R, Klingbiel D, et al. Prognostic role of KRAS and BRAF in stage II and III resected colon cancer: results of the translational study on the PETACC-3, EORTC 40993, SAKK 60-00 trial. 2010. J Clin Oncol. Jan 20; 28 (3): 466-74.

23. Kakar S, Deng G, Sahai V, Matsuzaki K, Tanaka H, Miura S. Clinicopathologic characteristics, CpG island methylator phenotype, and BRAF mutations in microsatellite-stable colorectal cancers without chromosomal instability. Arch Pathol Lab Med 2008; 132 (6): 958-64.

24. French AJ, Sargent DJ, Burgart LJ, Foster NR, Kabat BF, Goldberg R, et al. Prognostic significance of defective mismatch repair and BRAF V600E in patients with colon cancer. Clin Cancer Res 2008; 14 (11): 3408-15.

25. Stefanoff CG, Hassan R, González AC, Andrade LA, Tabak DG, Romano S, et al. Laboratory strategies for efficient handling of paraffin-embedded tissues for molecular detection of clonality in non-hodgkin lymphomas. Diagn Mol Pathol 2003; 12: 79-87.

26. Lahiri DK, Nurnberger JI Jr. A rapid non-enzymatic method for the preparation of HMW DNA from blood for RFLP studies. Nucleic Acids Res 1991; 19: 5444.

27. Boland CR, Thibodeau SN, Hamilton SR, Sidransky D, Eshleman JR, Burt RW, et al. A National Cancer Institute Workshop on Microsatellite Instability for cancer detection and familial predisposition: development of international criteria for the determination of microsatellite instability in colorectal cancer. Cancer Res 1998; 58: 5248-57.

28. Umar A, Boland CR, Terdiman JP, Syngal S, de la Chapelle A, Rüschoff J, et al. Revised Bethesda Guidelines for hereditary nonpolyposis colorectal cancer (Lynch syndrome) and microsatellite instability. J Natl Cancer Inst 2004; 96: 261-8.

29. Raptis S, Mrkonjic M, Green RC, Pethe VV, Monga N, Chan YM, et al. MLH1 -93G $>$ A promoter polymorphism and the risk of microsatellite-unstable colorectal cancer. J Natl Cancer Inst 2007; 99: 463-74.

30. Minoo P, Zlobec I, Peterson M, Terracciano L, Lugli A. Characterization of rectal, proximal and distal colon cancers based on clinicopathological, molecular and protein profiles. Int J Oncol 2010; 37: 707-18.

31. Roa I, Sánchez T, Majlis A, Schalper K. Mutación del gen KRAS en el cáncer de colon y recto. Rev Med Chile 2013; 141 (9): 1166-72.

32. Roa I, Game A, Bizama C, Schalper K. Mutación del gen BRAF en pacientes con cánceres de colon y recto con KRAS no mutado. Rev Med Chile 2014; 142 (1): 55-60. 
33. Zlobec I, Bihl MP, Schwarb H, Terracciano L, Lugli A. Clinicopathological and protein characterization of BRAF-and K-RAS-mutated colorectal cancer and implications for prognosis. Int J Cancer 2010; 127: 367-80.

34. Brim H, Mokarram P, Naghibalhossaini F, Saberi-Firoozi M, Al-Mandhari M, Al-Mawaly K, et al. Impact of BRAF, MLH1 on the incidence of microsatellite instability high colorectal cancer in populations based study. Mol Cancer 2008; 7: 68 .

35. Kato S, Iida S, Higuchi T, Ishikawa T, Takagi Y, Yasuno M, et al. PIK3CA mutation is predictive of poor survival in patients with colorectal cancer. Int J Cancer 2007; 121 : 1771-8.

36. Roth AD, Tejpar S, Delorenzi M, Yan P, Fiocca R, Klingbiel D, et al. Prognostic role of KRAS and BRAF in stage II and III resected colon cancer: results of the translational study on the PETACC-3, EORTC 40993, SAKK 60-00 trial. J Clin Oncol 2010; 28: 466-74.

37. Oliveira C, Velho S, Moutinho C, Ferreira A, Preto A, Domingo E, et al. KRAS and BRAF oncogenic mutations in MSS colorectal carcinoma progression. Oncogene 2007; 26: 158-63.

38. Boparai KS, Dekker E, Polak MM, Musler AR, van Eeden $S$, van Noesel CJ. A serrated colorectal cancer pathway predominates over the classic WNT pathway in patients with hyperplastic polyposis syndrome. Am J Pathol 2011; 178: 2700-7.

39. Prahallad A, Sun C, Huang S, Di Nicolantonio F, Salazar $\mathrm{R}$, Zecchin D, et al. Unresponsiveness of colon cancer to BRAF (V600E) inhibition through feedback activation of EGFR. Nature 2012; 483: 100-3.

40. Oliveira C, Westra JL, Arango D, Ollikainen M, Domingo E, Ferreira A, et al. Distinct patterns of KRAS mutations in colorectal carcinomas according to germline mismatch repair defects and hMLH1 methylation status. Hum Mol Genet 2004; 13: 2303-11.

41. Tejpar S, Celik I, Schlichting M, Sartorius U, Bokemeyer C, Van Cutsem E. Association of KRAS G13D tumor mutations with outcome in patients with metastatic colorectal cancer treated with first-line chemotherapy with or without cetuximab. J Clin Oncol 2012; 30: 3570-7.

42. Bazan V, Agnese V, Corsale S, Calò V, Valerio MR, Latteri MA, et al. Specific TP53 and/or Ki-ras mutations as independent predictors of clinical outcome in sporadic colorectal adenocarcinomas: results of a 5-year Gruppo Oncologico dell'Italia Meridionale (GOIM) prospective study. Ann Oncol 2005; 16: 50-5.

43. Heinemann V, Douillard JY, Ducreux M, Peeters M. Targeted therapy in metastatic colorectal cancer-an example of personalised medicine in action. Cancer Treat Rev
2013; 39: 592-601.

44. Modest DP, Stintzing S, Laubender RP, Neumann J, Jung A, Giessen C, et al. Clinical characterization of patients with metastatic colorectal cancer depending on the KRAS status. Anticancer Drugs 2011; 22: 913-8.

45. Jancík S, Drábek J, Radzioch D, Hajdúch M. Clinical relevance of KRAS in human cancers. J Biomed Biotechnol 2010; 150960.

46. Davies H, Bignell GR, Cox C, Sephens P, Edkins S, Clegg $\mathrm{S}$, et al. Mutations of the BRAF gene in human cancer. Nature 2002; 417 (6892): 949-54.

47. Bader AG, Kang S, Zhao L, Vogt PK. Oncogenic PI3K deregulates transcription and translation. Nat Rev Cancer 2005; 5 (12): 921-9. Review.

48. Samuels Y, Wang Z, Bardelli A, Silliman N, Ptak J, Szabo $S$, et al. High frequency of mutations of the PIK3CA gene in human cancers. Science 2004; 304: 554.

49. Velho S, Oliveira C, Ferreira A, Ferreira AC, Suriano G, Schwartz S Jr, et al. The prevalence of PIK3CA mutations in gastric and colon cancer. Eur J Cancer 2005; 41: 1649-54.

50. Velho S, Moutinho C, Cirnes L, Albuquerque C, Hamelin $\mathrm{R}$, Schmitt F, et al. BRAF, KRAS and PIK3CA mutations in colorectal serrated polyps and cancer: primary or secondary genetic events in colorectal carcinogenesis? BMC Cancer 2008; 8: 255.

51. Liao X, et al. Aspirin use, tumor PIK3CA mutation, and colorectal-cancer survival. N Engl J Med 2012; 367 (17): 1596-606.

52. Nishihara R., Lochhead P, Kuchiba A, Jung S, Yamauchi $\mathrm{M}$, Liao X, et al. Aspirin use and risk of colorectal cancer according to BRAF mutation status. JAMA 2013; 309 (24): 2563-71.

53. Amatu A, Bencardino K, Sartore-Bianchi A, Siena S. Aspirin for colorectal cancer with PIK3CA mutations: the rising of the oldest targeted therapy? Ann Transl Med 2013; 1 (2): 12.

54. Sinicrope FA, Sargent DJ. Molecular pathways: microsatellite instability in colorectal cancer: prognostic, predictive, and therapeutic implications. Clin Cancer Res 2012; 18: 1506-12.

55. Ribic CM, Sargent DJ, Moore MJ, Thibodeau SN, French AJ, Goldberg RM, et al. Tumor microsatellite-instability status as a predictor of benefit from fluorouracil-based adjuvant chemotherapy for colon cancer. N Engl J Med 2003; 349: 247-57.

56. Vilar E, Scaltriti M, Balmaña J, Saura C, Guzmán M, Arribas J, et al. Microsatellite instability due to hMLH1 deficiency is associated with increased cytotoxicity to irinotecan in human colorectal cancer cell lines. Br J Cancer 2008; 99: 1607-12. 